\title{
Operational experience of a Solar Power Plant with a Dual-Axis Solar Tracking System in the conditions of the Southern Urals
}

\author{
S. V. Mitrofanov ${ }^{1, *}, D . K$. Baykasenov ${ }^{1}$, and A. U. Nemaltsev ${ }^{1}$ \\ ${ }^{1}$ Orenburg State University, Orenburg, Russia
}

\begin{abstract}
The paper substantiates the need for the introduction of solar tracking systems for photovoltaic plants and presents a brief review of the scientific and technical literature on the development of solar trackers. Principal scheme of a heliostation with a dual-axis solar tracker of Orenburg State University has been designed. A comparative analysis of the generated electricity by a statically located solar module and a solar module with the dual-axis solar tracker is represented. Data for analysis have been obtained in Orenburg (Russia) using the developed automated system for remote diagnostics and monitoring of photovoltaic system parameters and a wireless weather station HP2000. The use of a dual-axis heliotracker for the solar plant in the conditions of the Southern Urals has allowed to increase the generation of electricity by $34.7 \%$ compared to a statically located PV panel.
\end{abstract}

\section{Introduction}

Currently, the cheapest source of energy is fossil fuels (for example, coal). In turn, the burning of coal in thermal power plants leads to the release of significant amount of greenhouse gases (mostly carbon dioxide $\mathrm{CO} 2$ ). They affect the climate and cause to global warming due to heating the atmosphere of our planet. For this reason, the world community decided to develop measures to reduce the negative impact of greenhouse gas emissions [1].

The first agreement on environmental protection is the Kyoto Protocol [2], which allows trading quotas on greenhouse gas emissions.

The most effective mean of reducing greenhouse gas emissions is the use of renewable energy sources (RES), such as solar (SPP) and wind (WPP) power plants $[3,4]$. In the Russian Federation, RES are progressed due to photovoltaic stations, because solar energy is inexhaustible, distributed everywhere and is environmentally friendly in comparison with traditional sources. [5]. In addition, the development of solar energy in recent years has been promoted due to the reduction the cost of PV panels. In Russia the generation of electricity based on SPP has increased by $35 \%$, which amounted to 758.4 million $\mathrm{kWh}$ in 2018 (compared with 2017). The part of WPP, SPP in total generation will increase to $1.8 \%$ in 2024 . It is planned to introduce new SPP with a total capacity of 2851 MW during 20192024.

Now the total capacity of the SPP of the Orenburg region is $225 \mathrm{MW}$. Also in the region it is planned to construct five PV plants with the total capacity of 120 MW (table 1).
Table 1. Solar power plants planned for construction in the Orenburg region.

\begin{tabular}{|c|c|}
\hline Station name & Power (W) \\
\hline Chebenkovskaya SPP & 45 \\
\hline Elshanskaya SPP & 25 \\
\hline Dombarovskaya SPP & 25 \\
\hline Shildinskaya SPP & 15 \\
\hline Grigorievskaya SPP & 10 \\
\hline
\end{tabular}

The sun moves during the day from east to west, and since the stationary panels are oriented to the south, therefore, the highest efficiency of the SPP is at noon. However, considerable power is available in the morning and in the afternoon. Increasing the difference between the angles of falling light flux on the surface of solar batteries (SB) and the normal of solar modules leads to a decrease in the power that SPP generates [6].

The solar tracking system is applied to increase the efficiency of the SPP. The main components of the solar tracker: microcontroller, guidance system to the sun and motors. The sun guidance system is based either on light dependent resistor (LDR) [7-9] or on GPS module $[10,11]$.

The purpose of this article is to conduct a comparative analysis of the power generated by the statically located solar panel and the PV module with a dual-axis solar tracking system in the conditions of the Southern Urals.

Research problems:

- Designing a principal scheme of a solar power plant with a dual-axis solar tracking system;

- Development of an automated system for remote diagnostics and control solar plant parameters (ASRDCSPP);

\footnotetext{
*Corresponding author: mitser2002@mail.ru
} 
- Materials and methods;

- Results and discussions.

\section{Designing a principal scheme of a solar power plant with a dual-axis solar tracking system}

SPP with the statically located PV panel and the solar module with a dual-axis tracker have been installed on the roof of the building of the Faculty of Electrical Power Engineering. The total power of the station is 800 $\mathrm{W}[12]$.

The electrical circuit of the PV plant is shown in Figure 1. Load redundancy system is provided: when battery charge below the setpoint, the PWM charge controller sends a command to turn off the KV1 relay. At the same time, power is supplied to the KV2 relay and the contact KV3.1 relay switches the power supply of load to the town mains (figure 1) [13].

The solar tracking system is based on LDR [14,15]. During the working of the SPP, it has been revealed that in the winter in the Orenburg region, snow and ice are formed on the LDR's case, thus it leads to incorrect operation of the solar tracking system. Thereby to improve the reliability of the heliotracker and minimize the error of pointing to the sun, we need to use a tracking system that works on GPS [16].

SPP protection against wind gusts and snow sticking to PV panels has been implemented [17-19].

\section{Development of an automated system for remote diagnostics and control solar plant parameters}

The development of the ASRDCSPP is associated with the lack of databases (DB) about the generation of SPP electricity in the Orenburg region, which is a prospective area for the development of solar energy [20,21].

Block diagram shows the principle of operation of the ASRDCSPP (figure 2). Readings from sensors are recorded on a memory card. In parallel, Arduino transmits this data via Bluetooth to a smartphone. The latter transmits readings from the sensors to the cloud storage, where the data are displayed in graphical and tabular forms. An online laboratory has been developed, it is a website with all the graphics of the ASRDCSPP (voltage on $\mathrm{SB}$, charging rate, power generated by each $\mathrm{PV}$ module as a function of time).

\section{Materials and methods}

The purpose of the experiment is to compare power generated by the statically located solar panels and the PV module with the dual-axis solar tracker. The experiment was conducted on June 6, 2019 in Orenburg (141, Pobeda Avenue). During the day in online mode complex had formed a database for the generation of electricity, which served as the basis for conducting this research. To determine the level of solar insolation and

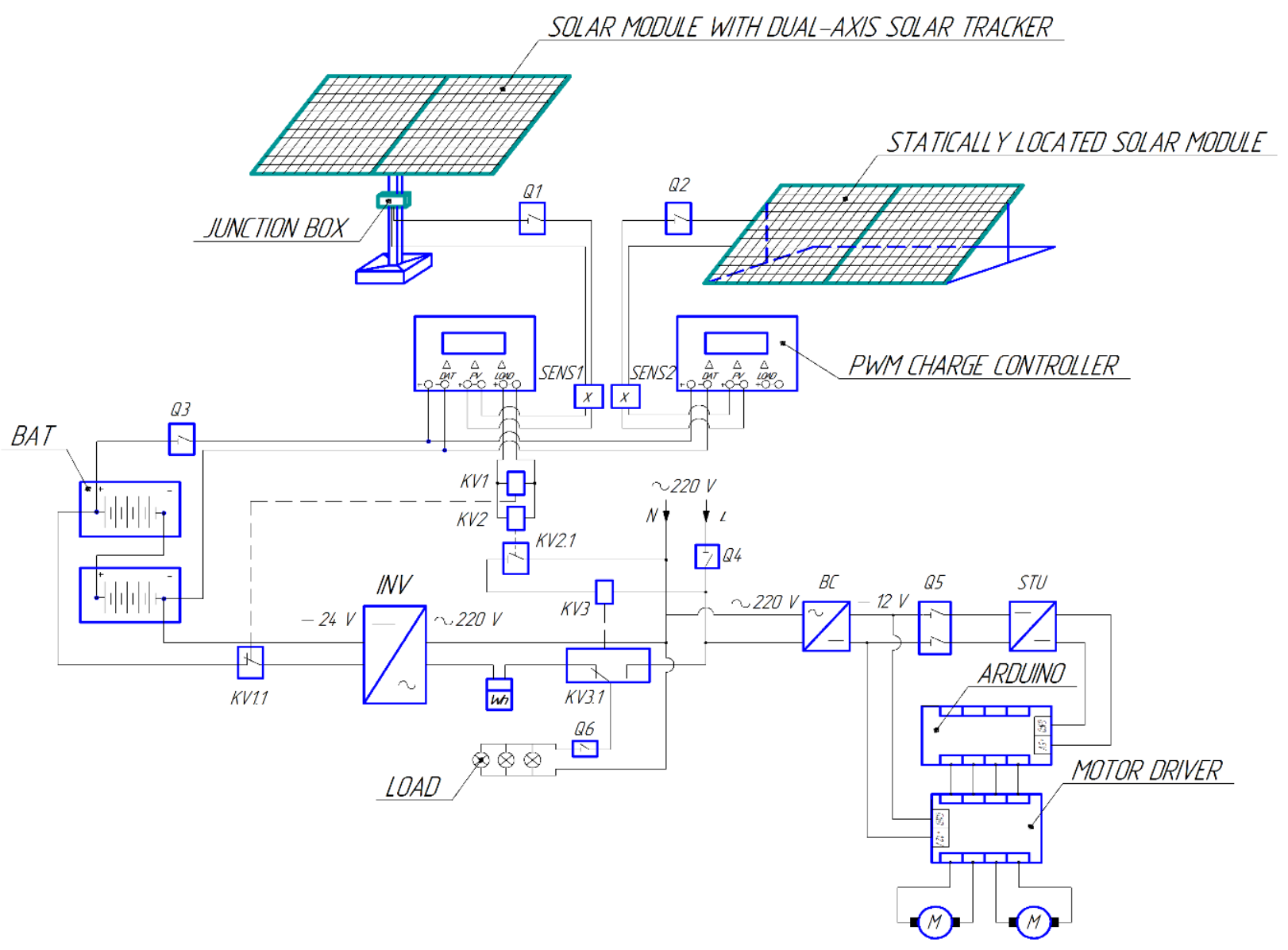

Fig. 1. Principal scheme of a solar power plant of the Orenburg State University. 


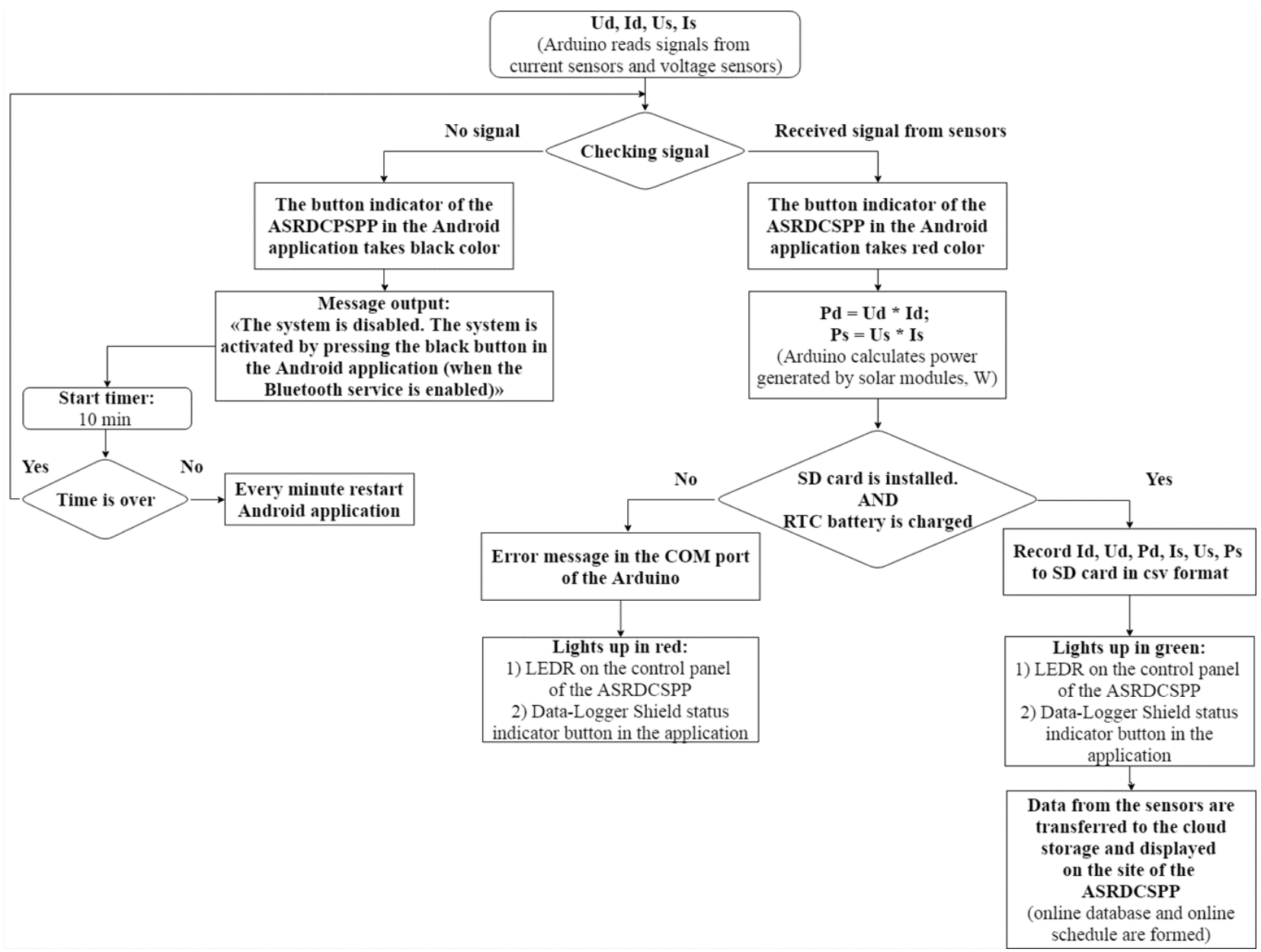

Fig. 2. The algorithm of an automated system for remote diagnostics and control solar plant parameters.

assess weather conditions, the Wi-Fi weather station HP2000 was installed on the platform of the solar tracker. Analysis of weather data had revealed that partly cloudy conditions prevailed in Orenburg on June 6, the sun rose at 05:02, the sunset was at 21:42, and the duration of this sunny day - 16 hours 39 minutes.

Batteries were used as load (24 V, $100 \mathrm{Ah})$. It should be noted that when we used this type of load and the batteries were fully charged, the sensors recorded the peak values of the generated power. It was associated with the operation of the PWM charge controller. To eliminate this factor, it is planned to introduce an active power consumer with an intelligent control system that will maximally load solar modules depending on a number of conditions.

\section{Results and discussions}

The power diagram clearly shows the process. PV panels began to generate power from 6:00 (figure 3). At 8:00 the statically located solar module generated $37 \mathrm{~W}$ and solar module with the tracker generated $96.7 \mathrm{~W}$. As the sun was rising, the generation was growing and at 10:00 was $160.52 \mathrm{~W}$ and $219.25 \mathrm{~W}$, respectively. Then, as the sun had risen, until 12:00, the power curve of the solar module with the tracker increased, and the power curve of the statically located SB from 10:00 to 12:00 had a falling character (CV mode). This is due to the fact that the battery charge is $90 \%$, while the PWM charge controller sets a limit on the magnitude of the charge current $(0.58 \mathrm{~A})$ and the voltage on the batteries $(27 \mathrm{~V})$ so that the batteries do not overcharge (figure 4). From 12:00 to $14: 00$, the weather station recorded a decrease in solar insolation from $445.1 \mathrm{~W} / \mathrm{m} 2$ to $243.3 \mathrm{~W} / \mathrm{m} 2$ (figure 5).

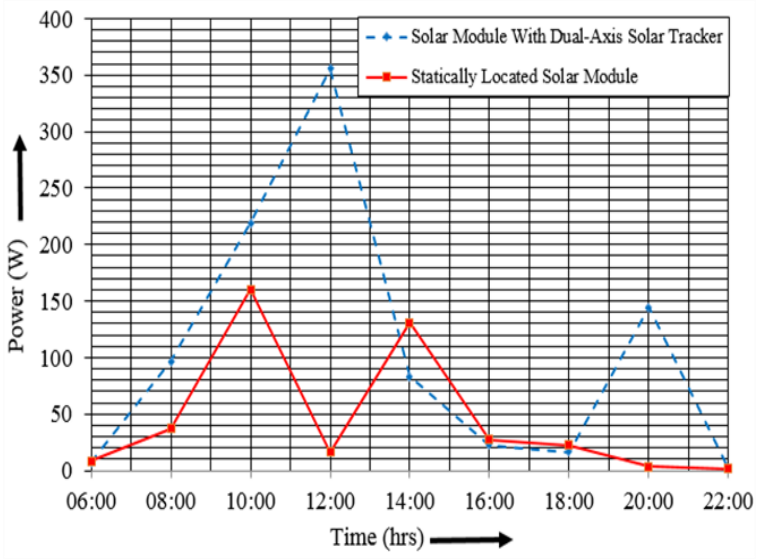

Fig. 3. Graphical dependence of the power generation of photovoltaic modules. 


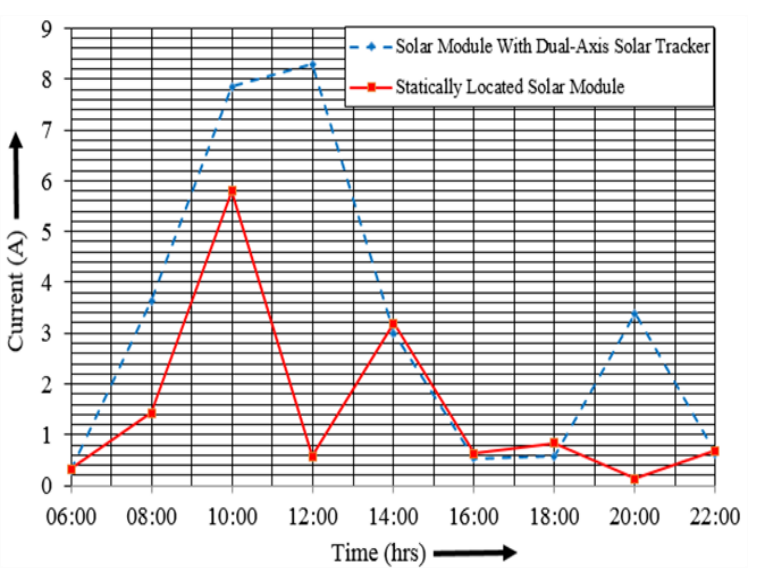

Fig. 4. Graphical dependence of the generation current of solar batteries.

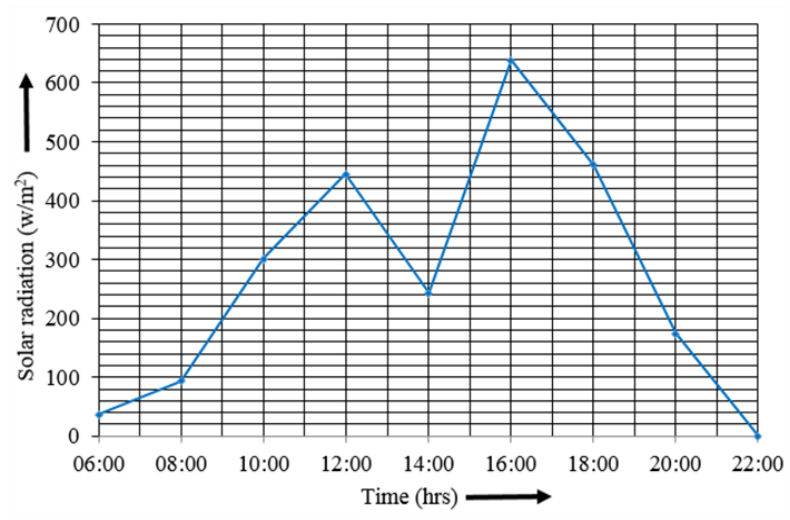

Fig. 5. Graphical dependence of the solar radiation.

Because of solar activity reduction, the amount of current generated by the solar module with the tracker decreased from 8.29 A to $3 \mathrm{~A}$, and from 14:00 the charge controller worked in current limiting mode. On the interval from 12:00 to 14:00 the battery charge controller of the stationary solar module operated in charging mode. From 14:00 to $16: 00$, the weather station recorded an increase in solar insolation, but by this time the batteries of the statically located PV module had charged up to $90 \%$ and the PWM controller had been operating in CV mode. At 16:00, the HP2000 registered the maximum daily insolation value - $637.9 \mathrm{~W} / \mathrm{m}^{2}$. Then, until 22:00 the battery charge controller of the statically located SB operated in CV mode. And from 18:00 to 20:00 for the solar module with the tracker - another battery charge cycle, which had ended with the CV mode until 22:00.

The average values of the energy generated by the solar module with a tracker and the statically located SB per day were $1567 \mathrm{Wh}$ and $1023 \mathrm{Wh}$ respectively. The use of the dual-axis solar tracker in summer day with variable cloudiness has allowed to increase electricity generation by $34.7 \%$.

As part of the research work, it was revealed that, due to the peculiarities of the SPP operation, there was no possibility to estimate the maximum power that two types of solar modules could generate. This is because of the fact the batteries (intermittent load) are the load of the solar plant now.

\section{Conclusions}

A principal scheme of a solar power plant has been developed, which provides load reservation and separate electricity metering from two solar modules. A complex has been developed and it allows to form a DB about the generation of SPP electricity, as well as remotely monitor plant operation parameters via the web site or an Android application. The efficiency of using dual-axis solar tracker in Orenburg has been experimentally proven. The increase in electricity generation using the dual-axis tracker is $34.7 \%$ relative to the statically located PV module.

\section{References}

[1] A. Rezvani, M. Gandomkar, M. Izadbakhsh, and A. Ahmadi, Environmental/economic scheduling of a micro-grid with renewable energy resources J. Cleaner Prod. 87, 216-26 (2015)

[2] L.Viguier, M. Babiker, J. Reilly, 2003 The costs of the Kyoto Protocol in the European Union J. Energy Pol. 31, 459-81

[3] C. Breyer, D. Bogdanov, A. Gulagi, A. Aghahosseini, L. Barbosa, O. Koskinen, M. Barasa, U. Caldera, S. Afanasyeva, M. Child, J. Farfan, P. Vainikka, On the role of solar photovoltaics in global energy transition scenarios J. Prog. Photovoltaics 25, 727-45 (2017)

[4] F. Corcelli, M. Ripa, S. Ulgiati, End-of-life treatment of crystalline silicon photovoltaic panels. An emergy-based case study J. Cleaner Prod. 161, 1129-42 (2017)

[5] S.V. Mitrofanov, D.K. Baykasenov, M.A. Suleev, Simulation Model of Autonomous Solar Power Plant with Dual-Axis Solar Tracker Int. Ural Conf. on Green Energy (Chelyabinsk: IEEE) 90-96 (2018)

[6] M.M. Rahman, M. Hasanuzzaman, N.A. Rahim, Effects of operational conditions on the energy efficiency of photovoltaic modules operating in Malaysia J. Cleaner Prod. 143, 912-24 (2017)

[7] M. Ghassoul, Single axis automatic tracking system based on Pilot scheme to control the solar panel to optimize solar energy extraction J. Energy Reports. 4, 520-27 (2018)

[8] F.M. Hoffmann, R.F. Molz, J.V. Kothe, E.O.B. Nara, L.P.C. Tedesco, Monthly profile analysis based on a two-axis solar tracker proposal for photovoltaic panels J. Renewable Energy 115, 750-59 (2018)

[9] H. Bentaher, H. Kaich, N. Ayadi, M. Ben Hmouda, A. Maalej, U. Lemmer, A simple tracking system to monitor solar PV panels J. Energy Conversion and Management 78, 872-75 (2014)

[10] J. Song, Y. Zhu, D. Xia, Y. Yang, A photovoltaic solar tracking system with bidirectional sliding axle for building integration J. Energy Procedia 61, 1638-41 (2014) 
[11] M.H.M. Sidek, N. Azis, W.Z.W. Hasan, M.Z.A.Ab. Kadir, S. Shafie, M.A.M. Radzi, Automated positioning dual-axis solar tracking system with precision elevation and azimuth angle control J. Energy 124, 160-70 (2017)

[12] S.V. Mitrofanov, D.K. Baykasenov, A.U. Nemaltsev, Solar power plants with sun-tracking systems The University complex as a regional center of education, science and culture (Orenburg: OSU) 296670 (2017)

[13] S.V. Mitrofanov, A.U. Nemaltsev, D.K. Baykasenov, Primary testing of automated dual-axis solar tracker in the climatic conditions of the Orenburg region as the prospects for the establishment of a hardware-software complex J. ISJAEE 7-9, 43-54 (2018)

[14] S.V. Mitrofanov, A.V. Potekhenchenko, A.U. Nemaltsev, Development of the control system of a solar tracker based on the Arduino microcontroller The University complex as a regional center of education, science and culture (Orenburg: OSU) 470-73 (2017)

[15] S.V. Mitrofanov, A.V. Potekhenchenko, A.U. Nemaltsev, Using microcontrollers in a solar tracking control system The University complex as a regional center of education, science and culture (Orenburg: OSU) 345-47 (2017)

[16] S.V. Mitrofanov, D.K. Baykasenov, M.A. Suleev, Modernized control system of automated dualaxis solar tracker in Orenburg region Proc. 8th Nation. Conf. The Power engineering: state, problems, prospects (Orenburg: OSU) 318-19 (2018)

[17] S.V. Mitrofanov, D.K. Baykasenov, M.A. Suleev, Complex System of Protection of Autonomous Solar Power Plant with Sun-Tracking System Int. Conf. on ICIEAM (Moscow: IEEE) 1-5 (2018)

[18] S.V. Mitrofanov, A.V. Potekhenchenko, Wind protection for solar power plant The University complex as a regional center of education, science and culture (Orenburg: OSU) 466-69 (2017)

[19] S.V. Mitrofanov, A.V. Potekhenchenko, Method of protection of solar power station against accumulation of snow and ice The University complex as a regional center of education, science and culture (Orenburg: OSU) 462-65 (2017)

[20] D.K. Baykasenov, A.U. Nemaltsev, Development of hardware-software complex for solar power plant with dual-axis solar tracker Proc. Nation. Conf. Achievements of university science: from theory to practice (Orenburg: OSU) 43-46 (2018)

[21] S.V. Mitrofanov, D.K. Baykasenov, A.U. Nemaltsev, Designing of system for monitoring of solar power plant parameters with a dual-axis solar tracker The University complex as a regional center of education, science and culture (Orenburg: OSU) 348789 (2019)

[22] S.V. Mitrofanov, D.K. Baykasenov, A.U. Nemaltsev, Development of Automated System for Remote Control and Diagnostics of Solar Power Plant Parameters with Dual-Axis Solar Tracker Int. Conf. on ICIEAM (Sochi: IEEE) 1-5 (2019) 